\author{
ASTRODYNAMICS (C1.) \\ Mission and Constellation Design (8.) \\ Exclude Poster: No \\ Student (student: yes - sponsor: University of Glasgow)
}

Author: Ms Christie Maddock

University of Glasgow, Glasgow, United Kingdom, c.maddock@aero.gla.ac.uk

Mr Joan Pau Sanchez Cuartielles

University of Glasgow, Glasgow, United Kingdom, psanchez@eng.gla.ac.uk

Dr Massimiliano Vasile

University of Glasgow, Glasgow, United Kingdom, mvasile@aero.gla.ac.uk

Dr. Gianmarco Radice

University of Glasgow, Glasgow, United Kingdom, gradice@aero.gla.ac.uk

\title{
COMPARISON OF SINGLE AND MULTI-SPACECRAFT CONFIGURATIONS FOR NEA DEFLECTION BY SOLAR SUBLIMATION
}

\begin{abstract}
Since the first Near Earth Object (NEO) defence system, Project Icarus, was published in 1967, where the driving factor was a very short anticipating time (i.e. time available to act on the asteroid), the Near Earth Asteroid (NEA) hazard outlook has changed drastically. In the current state of NEO research, long-term missions are becoming more realistic and as such, the options for low thrust systems are being investigated as a viable option for deviating the asteroid path.

Surface ablation approaches have been previously proposed using several techniques such as lasers and nuclear explosives. The method presented here hinges on directing solar energy using mirrors onto a small area on the surface of the asteroid. This concentrated heat then sublimates the surface matter creating narrow but expanding jets of gas and dust that produce a low continuous thrust. This low thrust would then alter the orbit of the NEA by producing a change in velocity, similar to the effect of the 'tail' on a comet.

This paper adds a new important trade-off to the problem: a comparison between a single structure and a multi-mirror system. The systems analysed include both single and dual mirror configurations, for both a single spacecraft and multiple spacecraft in formation. The criteria include ease of launch, reliability, flexibility in achieving the mission objective, in-space mass and a basic cost analysis.

The concept, and potential benefits, of formation flying have long been known. The barrier however, has been the high level of complexity involved in the control of the individual spacecraft within the formation. Advances in control algorithms and associated technologies have opened the door to using spacecraft formations for specialized missions, such as small-body missions which operate in highly perturbed environments.

This paper compares the complexities of deploying and operating a large rigid structure around asteroids, with the control of a swarm of smaller structures. Configurations are presented for different NEAs, time-in-advance, and achieved deflections.
\end{abstract}

